\title{
Spectral Method with the Tensor-Product Nodal Basis for the Steklov Eigenvalue Problem
}

\author{
Xuqing Zhang, Yidu Yang, and Hai Bi \\ School of Mathematics and Computer Science, Guizhou Normal University, Guiyang 550001, China \\ Correspondence should be addressed to Hai Bi; bihaimath@gznu.edu.cn
}

Received 18 January 2013; Accepted 3 September 2013

Academic Editor: Daoyi Dong

Copyright ( 2013 Xuqing Zhang et al. This is an open access article distributed under the Creative Commons Attribution License, which permits unrestricted use, distribution, and reproduction in any medium, provided the original work is properly cited.

\begin{abstract}
This paper discusses spectral method with the tensor-product nodal basis at the Legendre-Gauss-Lobatto points for solving the Steklov eigenvalue problem. A priori error estimates of spectral method are discussed, and based on the work of Melenk and Wohlmuth (2001), a posterior error estimator of the residual type is given and analyzed. In addition, this paper combines the shifted-inverse iterative method and spectral method to establish an efficient scheme. Finally, numerical experiments with MATLAB program are reported.
\end{abstract}

\section{Introduction}

Since Steklov eigenvalue problems have important physical background and many applications, for instance, they appear in the analysis of stability of mechanical oscillators immersed in a viscous fluid (see [1] and the references therein), in the analysis of the antiplane shearing on a system of collinear faults under slip-dependent friction law (see [2]), in the study of surface waves (see [3]), in the study of the vibration modes of a structure in contact with an incompressible fluid (see [4]) and vibration of a pendulum (see [5]), and in the eigenoscillations of mechanical systems with boundary conditions containing frequency [6], and thus, numerical methods for solving Steklov eigenvalue problems have received increasing attention in recent years. Bramble and Osborn [7] studied the Galerkin approximation of a Steklov eigenvalue problem of nonself-adjoint second order elliptic operators in smooth domain, Andreev and Todorov [8] discussed the isoparametric finite element method for the approximation of the Steklov eigenvalue problem of second-order self-adjoint elliptic differential operators, Armentano and Padra [9] introduced and analyzed the conforming linear finite element approximation of the Steklov eigenvalue problem in a bounded polygonal domain, Alonso and Russo [10], Yang et al. [11], and Li et al. [12] studied nonconforming finite elements approximation of the Steklov eigenvalue problem, Li and Yang [13] and Bi and
Yang [14] discussed a two-grid method of the conforming and non-conforming finite element method, respectively, Li et al. [15] studied the extrapolation and superconvergence of the Steklov eigenvalue problem, Tang et al. [16] studied the boundary element approximation, and Cao et al. [17] discussed multiscale asymptotic method for Steklov eigenvalue equations in composite medias. However, to the best of our knowledge, there have been no reports on spectral method for Steklov eigenvalue problems.

Spectral method is an important numerical method for solving differential equations developed after finite-difference and finite-element methods. Because spectral method has the characteristics of superior accuracy, it is widely used in the field of meteorology, physics, and mechanics (see $[18,19])$. This paper discusses spectral method with the tensor-product nodal basis at the Legendre-Gauss-Lobatto (LGL for short) points for solving the Steklov eigenvalue problem. We use spectral approximation theory (see [20]) to give a priori error estimates. Based on the work of [21], we discuss a posterior error estimator of the residual type. Moreover, inspired by the work of [22] this paper combines the shifted-inverse iterative method and spectral method to propose an efficient computation scheme. Finally, by using program with Matlab, we implement the numerical experiments and get satisfactory results. 
The rest of the paper is organized as follows. In Section 2, some preliminaries needed in this paper are presented. In Section 3, we discuss a priori and a posterior error estimates for spectral method. In Section 4, we establish an efficient scheme combining the shifted-inverse iteration and spectral method. In Section 5, the numerical experiments on the square domain are reported.

\section{Preliminaries}

We consider the following boundary value problem:

$$
\begin{gathered}
-\Delta u+u=0, \quad \text { in } \Omega, \\
\frac{\partial u}{\partial n}=f, \quad \text { on } \partial \Omega,
\end{gathered}
$$

where $\Omega \subset R^{2}$ is a rectangular domain and $\partial / \partial n$ is the outward normal derivative on $\partial \Omega$. We denote the Sobolev space with norm $\|\cdot\|_{m}$ and seminorm $|\cdot|_{m}$ by $H^{m}(\Omega)$. Let $\|\cdot\|_{0}$ and $\|\cdot\|_{0, \partial \Omega}$ be the norms in the space $L^{2}(\Omega)$ and $L^{2}(\partial \Omega)$, respectively.

Throughout this paper, $C$ denotes a generic positive constant independent of the polynomial degrees $N$, which may not be the same at each occurrence.

The variational problem associated with (1) is given by the following.

Find $u \in H^{1}(\Omega)$, such that

$$
a(u, v)=b(f, v), \quad \forall v \in H^{1}(\Omega),
$$

where

$$
\begin{gathered}
a(u, v)=\int_{\Omega} \nabla u \cdot \nabla v+\int_{\Omega} u v, \quad \forall u, v \in H^{1}(\Omega), \\
b(f, v)=\int_{\partial \Omega} f v, \quad \forall f, v \in L^{2}(\partial \Omega) .
\end{gathered}
$$

Obviously, the bilinear form $a(\cdot, \cdot)$ is continuous and $H^{1}(\Omega)$ elliptic, that is, there exists constants $A$ and $B$ independent of $u, v$, such that

$$
\begin{gathered}
|a(u, v)| \leq A\|u\|_{1}\|v\|_{1}, \quad \forall u, v \in H^{1}(\Omega), \\
a(v, v) \geq B\|v\|_{1}^{2}, \quad \forall u, v \in H^{1}(\Omega) .
\end{gathered}
$$

From the Lax-Milgram theorem we know that there exists a unique solution to (2).

The following regularity results (see [4]) will be needed in the sequel: for $f \in L^{2}(\partial \Omega)$, the solution of problem (1) $u \in H^{1+r / 2}(\Omega)$, and

$$
\|u\|_{1+r / 2} \leq M_{1}\|f\|_{0, \partial \Omega}
$$

furthermore, if $f \in H^{1 / 2}(\partial \Omega)$, then $u \in H^{1+r}(\Omega)$ and

$$
\|u\|_{1+r} \leq M_{2}\|f\|_{1 / 2, \partial \Omega}
$$

where $M_{1}, M_{2}$ are constants independent of $f$, and $r=1$ if $\Omega$ is convex and $r<\pi / \omega$ if $\Omega$ is concave (with $\omega$ being the largest inner angle of $\Omega$ ).
Define

$$
S_{N}(\Omega)=\operatorname{span}\left\{x^{i} y^{j} \mid 0 \leq i, j \leq N\right\} .
$$

The spectral-Galerkin approximation to (2) is the following.

Find $u_{N} \in S_{N}(\Omega)$, such that

$$
a\left(u_{N}, v_{N}\right)=b\left(f, v_{N}\right), \quad \forall v_{N} \in S_{N}(\Omega) .
$$

For $m \geq 3 / 2$ and each integer $N \geq 1$, we define the interpolation operator

$$
I_{N}: H^{m}(\Omega) \longrightarrow S_{N}(\Omega)
$$

with the LGL interpolation nodes in $\Omega$. We quote from [18] the following interpolation estimates for spectral method at the tensor-product LGL points

$$
\left\|u-I_{N} u\right\|_{1} \leq C(m) N^{1-m}\|u\|_{m} .
$$

Let $u \in H^{m}(\Omega)$ and $u_{N}$ be the solution of (2) and (8), respectively. Then we derive from Céa Lemma that

$$
\left\|u_{N}-u\right\|_{1} \leq C(m) N^{1-m}\|u\|_{m} .
$$

Notice that (11) is also valid for special method with modal basis (see $[18,19])$.

Using Aubin-Nitsche technique, we deduce the a priori error estimate from (5) and (10)-(11):

$$
\left\|u_{N}-u\right\|_{0, \partial \Omega} \leq C N^{1-m-r / 2}\|u\|_{m} .
$$

\section{Spectral Method and Its Error Estimates for the Steklov Eigenvalue Problem}

3.1. Spectral Method for the Steklov Eigenvalue Problem. We consider the following Steklov eigenvalue problem:

$$
\begin{gathered}
-\Delta u+u=0, \quad \text { in } \Omega, \\
\frac{\partial u}{\partial n}=\lambda u, \quad \text { on } \partial \Omega,
\end{gathered}
$$

where $\Omega \subset R^{2}$ is a rectangular domain.

The variational problem associated with (13) is given by the following.

Find $\lambda \in R, 0 \neq u \in H^{1}(\Omega)$, such that

$$
\begin{gathered}
a(u, v)=\lambda b(u, v), \quad \forall v \in H^{1}(\Omega), \\
\|u\|_{0, \partial \Omega}=1 .
\end{gathered}
$$

Let $(\lambda, u)$ be an eigenpair of (14); then from (6) we know that $u \in H^{1+r}(\Omega)$. follows.

The spectral-Galerkin approximation to (14) is as the

Find $\lambda_{N} \in R, 0 \neq u_{N} \in S_{N}(\Omega)$, such that

$$
\begin{gathered}
a\left(u_{N}, v_{N}\right)=\lambda_{N} b\left(u_{N}, v_{N}\right), \quad \forall v_{N} \in S_{N}(\Omega), \\
\left\|u_{N}\right\|_{0, \partial \Omega}=1 .
\end{gathered}
$$


Let $T: L^{2}(\partial \Omega) \rightarrow H^{1}(\Omega)$ and $T_{N}: L^{2}(\partial \Omega) \rightarrow S_{N}(\Omega)$ be the solution operators defined by

$$
\begin{gathered}
a(T f, v)=b(f, v), \quad \forall v \in H^{1}(\Omega), \\
a\left(T_{N} f, v\right)=b(f, v), \quad \forall v_{N} \in S_{N}(\Omega) .
\end{gathered}
$$

Obviously, $T: H^{1}(\Omega) \rightarrow H^{1}(\Omega)$ is completely continuous and $T_{N}: H^{1}(\Omega) \rightarrow S_{N}(\Omega)$ is a finite ranked operator. Equations (14) and (15) have the following equivalent operator forms, respectively:

$$
\begin{gathered}
T u=\lambda^{-1} u, \\
T_{N} u_{N}=\lambda_{N}^{-1} u_{N} .
\end{gathered}
$$

3.2. A Prior Error Estimates of Spectral Method for the Steklov Eigenvalue Problem. Let $(\lambda, u)$ be an eigenpair of (14), let $V(\lambda)$ be the space spanned by all eigenfunctions corresponding to the eigenvalue $\lambda$, and let $V_{N}(\lambda)$ be the direct sum of the eigenspace corresponding to the eigenvalues $\lambda_{N}$ of (15) that converge to $\lambda$.

Denote

$$
\begin{gathered}
\varepsilon_{N}=\varepsilon_{N}(\lambda)=\sup _{u \in V(\lambda),\|u\|_{1}=1} \inf _{v \in S_{N}(\Omega)}\|u-v\|_{1}, \\
\eta_{a}(N)=\sup _{f \in H^{1}(\Omega),\|f\|_{1}=1} \inf _{v \in S_{N}(\Omega)}\|T f-v\|_{1}, \\
\rho_{N}=\sup _{f \in L^{2}(\partial \Omega),\|f\|_{0, \partial \Omega}=1} \inf _{v \in S_{N}(\Omega)}\|T f-v\|_{1},
\end{gathered}
$$

where $\varepsilon_{N}$ characterizes the degree of the space $S_{N}(\Omega)$ approximating $V(\lambda), \eta_{a}(N)$ and $\rho_{N}$ characterize the degrees of $S_{N}(\Omega)$ approximating the generalized solution $T f$ of (2).

Lemma 1. Let $V(\lambda) \subset H^{m}(\Omega)$; then

$$
\begin{gathered}
\varepsilon_{N} \leq C N^{1-m} \sup _{u \in V(\lambda),\|u\|_{1}=1}\|u\|_{m}, \\
\eta_{a}(N) \leq C N^{-r}, \\
\rho_{N} \leq C N^{-r / 2} .
\end{gathered}
$$

Proof. We deduce from the interpolation estimate (10) that

$$
\begin{aligned}
\varepsilon_{N} & \leq \sup _{u \in V(\lambda),\|u\|_{1}=1}\left\|u-I_{N} u\right\|_{1} \\
& \leq C N^{1-m} \sup _{u \in V(\lambda),\|u\|_{1}=1}\|u\|_{m} .
\end{aligned}
$$

Using (10) and (6) we have

$$
\begin{aligned}
\eta_{a}(N) & \leq \sup _{f \in H^{1}(\Omega),\|f\|_{1}=1}\left\|T f-I_{N} T f\right\|_{1} \\
& \leq C N^{-r} \sup _{f \in H^{1}(\Omega),\|f\|_{1}=1}\|T f\|_{1+r} \\
& \leq C N^{-r} .
\end{aligned}
$$

From (10) and regularity estimate (5) we get

$$
\begin{aligned}
\rho_{N} & \leq \sup _{f \in L^{2}(\partial \Omega),\|f\|_{0, \partial \Omega}=1}\left\|T f-I_{N} T f\right\|_{1} \\
& \leq C N^{-r / 2} \sup _{f \in L^{2}(\partial \Omega),\|f\|_{0, \partial \Omega}=1}\|T f\|_{1+r / 2, \Omega} \\
& \leq C N^{-r / 2} .
\end{aligned}
$$

The proof is completed.

Theorem 2. Let $V(\lambda) \subset H^{m}(\Omega)$; then

$$
\lambda_{N}-\lambda \leq C\left\{N^{1-m} \sup _{u \in V(\lambda),\|u\|_{1}=1}\|u\|_{m}\right\}^{2} ;
$$

let $u_{N} \in V_{N}(\lambda)$ and $\left\|u_{N}\right\|_{1}=1$; then there exists $u \in V(\lambda)$, such that

$$
\begin{gathered}
\left\|u_{N}-u\right\|_{1} \leq C N^{1-m} \sup _{u \in V(\lambda),\|u\|_{1}=1}\|u\|_{m}, \\
\left\|u_{N}-u\right\|_{0, \partial \Omega} \leq C N^{1-m-r / 2} \sup _{u \in V(\lambda),\|u\|_{1}=1}\|u\|_{m} ;
\end{gathered}
$$

let $u \in V(\lambda)$ and $\|u\|_{1}=1$; then there exists $u_{N} \in V_{N}(\lambda)$, such that

$$
\left\|u_{N}-u\right\|_{1} \leq C N^{1-m} \sup _{u \in V(\lambda),\|u\|_{1}=1}\|u\|_{m} .
$$

Proof. From [20, 23] we have that

$$
\lambda_{N}-\lambda \leq C \varepsilon_{N}^{2}
$$

let $u_{N} \in V_{N}(\lambda)$ and $\left\|u_{N}\right\|_{1}=1$; then there exists $u \in V(\lambda)$, such that

$$
\begin{gathered}
\left\|u_{N}-u\right\|_{1} \leq C \varepsilon_{N}, \\
\left\|u_{N}-u\right\|_{0, \partial \Omega} \leq C \rho_{N} \varepsilon_{N} ;
\end{gathered}
$$

let $u \in V(\lambda)$ and $\|u\|_{1}=1$; then there exists $u_{N} \in V_{N}(\lambda)$, such that

$$
\left\|u_{N}-u\right\|_{1} \leq C \varepsilon_{N} .
$$

Combining Lemma 1 we obtain the desired results.

3.3. A Posterior Error Estimates for the Steklov Eigenvalue Problem. Theorem 2.1 in [24] gave the following results.

Lemma 3. There exists a positive constant $C$ and a linear bounded operator $I: L^{1}(\Omega) \rightarrow S_{N}(\Omega) \subset L^{1}(\Omega)$, such that for all $v \in H^{1}(\Omega)$ and an arbitrary edge e of $\partial \Omega$, and there holds

$$
\begin{gathered}
\|v-I v\|_{0} \leq C \frac{1}{N}\|\nabla v\|_{0}, \\
\|v-I v\|_{0, e} \leq C \frac{1}{\sqrt{N}}\|\nabla v\|_{0} .
\end{gathered}
$$


Analysis of the error indicators requires polynomial inverse estimates in weighted Sobolev spaces. Define on the interval $S=(0,1)$ the weight function $\Phi_{S}:=x(1-x)$; then there holds the following polynomial inverse estimates.

Lemma 4. Let $-1<\alpha<\beta, \sigma \in[0,1]$; then there exists $C_{1}$, $C_{2}=C(\alpha, \beta)$, and $C_{3}=C_{\sigma}$ such that for every univariate polynomial $\pi_{N}$ of degree $N$, there holds

$$
\begin{gathered}
\int_{S} \Phi_{S}(x)\left(\pi_{N}^{\prime}(x)\right)^{2} d x \leq C_{1} N^{2} \int_{S} \pi_{N}^{2}(x) d x \\
\int_{S} \Phi_{S}^{\alpha}(x) \pi_{N}^{2}(x) d x \leq C_{2} N^{2(\beta-\alpha)} \int_{S} \Phi_{S}^{\beta} \pi_{N}^{2}(x) d x \\
\int_{S} \Phi_{S}^{2 \sigma}(x)\left(\pi_{N}^{\prime}(x)\right)^{2} d x \leq C_{3} N^{2(2-\sigma)} \int_{S} \Phi_{S}^{\sigma} \pi_{N}^{2}(x) d x
\end{gathered}
$$

Proof. See Lemma 2.4 in [21].

Consider the domain $\kappa=(0,1)^{2}$ and the weight function $\Phi_{\kappa}:=\operatorname{dist}(x, \partial \kappa)$. From [21] we have the two-dimensional polynomial inverse estimates.

Lemma 5. Let $-1<\alpha<\beta, \sigma \in[0,1]$; then there exists $C_{1}$, $C_{2}=C(\alpha, \beta)$, and $C_{3}=C_{\sigma}$ such that for all polynomials $\pi_{N} \epsilon$ $S_{N}(\Omega)$ there hold

$$
\begin{array}{r}
\int_{\kappa} \Phi_{\kappa}\left|\nabla \pi_{N}\right|^{2} d x d y \leq C_{1} N^{2} \int_{\mathcal{\kappa}}\left|\pi_{N}\right|^{2} d x d y, \\
\int_{\kappa} \Phi_{\kappa}^{\alpha} \pi_{N}^{2} d x d y \leq C_{2} N^{2(\beta-\alpha)} \int_{\mathcal{\kappa}} \Phi_{\kappa}^{\beta} \pi_{N}^{2} d x d y, \\
\int_{\kappa} \Phi_{\kappa}^{2 \sigma}\left|\nabla \pi_{N}\right|^{2} d x d y \leq C_{3} N^{2(2-\sigma)} \int_{\kappa} \Phi_{\kappa}^{\sigma} \pi_{N}^{2} d x d y .
\end{array}
$$

Proof. See Lemma 2.5 in [21].

Lemma 6. Let $\alpha \in(1 / 2,1]$, set $\widehat{e}:=(0,1) \times\{0\}$, and let $\Phi_{\widehat{e}}$ be defined as the same as $\Phi_{S}$, that is, $\Phi_{\widehat{e}}=x(1-x)$. Then there exists $C_{\alpha}>0$, such that for every $\varepsilon \in(0,1)$ and every univariate polynomial $\pi_{N}$ of degree $N$, there exists an extension $v_{\widehat{e}} \in H^{1}(\kappa)$, such that the following holds:

$$
\begin{gathered}
\left.v_{\hat{e}}\right|_{\hat{e}}=\pi_{N} \cdot \Phi_{\widehat{e}}^{\alpha},\left.\quad v_{\widehat{e}}\right|_{\partial \kappa \mid \widehat{e}}=0, \\
\left\|v_{\widehat{e}}\right\|_{0, \kappa}^{2} \leq C_{\alpha} \varepsilon\left\|\pi_{N} \Phi_{\widehat{e}}^{\alpha / 2}\right\|_{0, \vec{e}}^{2} \\
\left\|\nabla v_{\widehat{e}}\right\|_{0, \kappa}^{2} \leq C_{\alpha}\left(\varepsilon N^{2(2-\alpha)}+\varepsilon^{-1}\right)\left\|\pi_{N} \Phi_{\widehat{e}}^{\alpha / 2}\right\|_{0, \hat{e}}^{2} .
\end{gathered}
$$

Proof. See Lemma 2.6 in [21].

Remark 7. Because there has an affine transformation from rectangular domain to the unit square, Lemma 5 is suited to rectangular domain, and Lemmas 4 and 6 are valid for all edges of rectangular.

Define the global error indicator $\eta_{\alpha}, \alpha \in[0,1]$, which is expressed as the sum of two terms:

$$
\eta_{\alpha}^{2}:=\eta_{\alpha ; B}^{2}+\eta_{\alpha ; E}^{2}
$$

where the first term $\eta_{\alpha ; B}^{2}$ is the weighted internal residual given by

$$
\eta_{\alpha ; B}^{2}:=\frac{1}{N^{2}}\left\|\left(\Delta u_{N}-u_{N}\right) \Phi_{\Omega}^{\alpha / 2}\right\|_{0}^{2},
$$

and the second term $\eta_{\alpha ; E}^{2}$ is the weighted edge residual given by

$$
\eta_{\alpha ; E}^{2}:=\frac{1}{N}\left\|\left(\lambda_{N} u_{N}-\frac{\partial u_{N}}{\partial n}\right) \Phi_{e}^{\alpha / 2}\right\|_{0, \partial \Omega}^{2},
$$

where the weight functions $\Phi_{\Omega}$ and $\Phi_{e}$ are scaled transformations of the weight functions $\Phi_{\kappa}$ and $\Phi_{\widehat{e}}$, respectively.

By using the proof of [21], we can get the following results.

Theorem 8. Let $\alpha \in[0,1]$. Then there exists $C>0$ independent of $N$, such that

$$
\left\|u-u_{N}\right\|_{1}^{2} \leq C\left\|\lambda u-\lambda_{N} u_{N}\right\|_{0, \partial \Omega}^{2}+C N^{2 \alpha}\left(\eta_{\alpha ; B}^{2}+\eta_{\alpha ; E}^{2}\right) .
$$

Proof. We denote $w:=u-u_{N}-I\left(u-u_{N}\right)$ where $I$ is an interpolation operator satisfying Lemma 3 . We derive from $H^{1}(\Omega)$-ellipticity of $a(\cdot, \cdot)$ that

$$
\begin{aligned}
C\left\|u-u_{N}\right\|_{1}^{2} \leq & a\left(u-u_{N}, u-u_{N}\right) \\
= & a\left(u-u_{N}, w\right)+a\left(u-u_{N}, I\left(u-u_{N}\right)\right) \\
= & \int_{\partial \Omega} \lambda u w-a\left(u_{N}, w\right) \\
& +\int_{\partial \Omega}\left(\lambda u-\lambda_{N} u_{N}\right) I\left(u-u_{N}\right) .
\end{aligned}
$$

Since

$$
\begin{aligned}
a\left(u_{N}, w\right) & =\int_{\Omega} \nabla u_{N} \cdot \nabla w+\int_{\Omega} u_{N} w \\
& =-\int_{\Omega} \Delta u_{N} w+\int_{\partial \Omega} \frac{\partial u_{N}}{\partial n} w+\int_{\Omega} u_{N} w,
\end{aligned}
$$

thus

$$
\begin{aligned}
C\left\|u-u_{N}\right\|_{1}^{2} \leq & \int_{\partial \Omega}\left(\lambda u-\lambda_{N} u_{N}\right) w+\int_{\partial \Omega}\left(\lambda_{N} u_{N}-\frac{\partial u_{N}}{\partial n}\right) w \\
& +\int_{\Omega}\left(\Delta u_{N}-u_{N}\right) w \\
& +\int_{\partial \Omega}\left(\lambda u-\lambda_{N} u_{N}\right) I\left(u-u_{N}\right) \\
= & \int_{\partial \Omega}\left(\lambda u-\lambda_{N} u_{N}\right)\left(u-u_{N}\right) \\
& +\int_{\partial \Omega}\left(\lambda_{N} u_{N}-\frac{\partial u_{N}}{\partial n}\right) w+\int_{\Omega}\left(\Delta u_{N}-u_{N}\right) w .
\end{aligned}
$$


By using Cauchy-Schwartz inequality, the trace theorem, and Lemma 3, we get

$$
\begin{gathered}
\int_{\partial \Omega}\left(\lambda u-\lambda_{N} u_{N}\right)\left(u-u_{N}\right) \leq\left\|\lambda u-\lambda_{N} u_{N}\right\|_{0, \partial \Omega}\left\|u-u_{N}\right\|_{1}, \\
\int_{\partial \Omega}\left(\lambda_{N} u_{N}-\frac{\partial u_{N}}{\partial n}\right) w \\
\leq C\left\|\lambda_{N} u_{N}-\frac{\partial u_{N}}{\partial n}\right\|_{0, \partial \Omega} \frac{1}{\sqrt{N}}\left\|\nabla\left(u-u_{N}\right)\right\|_{0}, \\
\int_{\Omega}\left(\Delta u_{N}-u_{N}\right) w \leq C\left\|\Delta u_{N}-u_{N}\right\|_{0} \frac{1}{N}\left\|\nabla\left(u-u_{N}\right)\right\|_{0} .
\end{gathered}
$$

Plugging (47) into (46) gives

$$
\begin{aligned}
C \| u- & u_{N} \|_{1} \\
\leq & \left\|\lambda u-\lambda_{N} u_{N}\right\|_{0, \partial \Omega}+C \frac{1}{\sqrt{N}}\left\|\lambda_{N} u_{N}-\frac{\partial u_{N}}{\partial n}\right\|_{0, \partial \Omega} \\
& +C \frac{1}{N}\left\|\Delta u_{N}-u_{N}\right\|_{0} .
\end{aligned}
$$

Setting $\alpha=0, \beta=\alpha$ in (32) and (35), respectively, we obtain $\eta_{0 ; B} \leq C N^{\alpha} \eta_{\alpha ; B}$ and $\eta_{0 ; E} \leq C N^{\alpha} \eta_{\alpha ; E}$, and thus

$$
C\left\|u-u_{N}\right\|_{1} \leq\left\|\lambda u-\lambda_{N} u_{N}\right\|_{0, \partial \Omega}+C N^{\alpha} \eta_{\alpha ; E}+C N^{\alpha} \eta_{\alpha ; B} .
$$

The proof is completed.

Lemma 9. Let $\alpha \in[0,1]$. Then there exists $C>0$ independent of $N$, such that

$$
\eta_{\alpha ; B}^{2} \leq C N^{2(1-\alpha)}\left\|u_{N}-u\right\|_{1}^{2}
$$

Proof. Denote $v_{\Omega}:=\left(u_{N}-\Delta u_{N}\right) \Phi_{\Omega}^{\alpha} \in H_{0}^{1}(\Omega), \alpha \in(0,1]$; then

$$
\begin{aligned}
\left\|v_{\Omega} \Phi_{\Omega}^{-\alpha / 2}\right\|_{0}^{2} & =\int_{\Omega}\left(u_{N}-\Delta u_{N}\right) v_{\Omega} \\
& =\int_{\Omega} u_{N} v_{\Omega}+\int_{\Omega} \nabla u_{N} \cdot \nabla v_{\Omega}-\int_{\partial \Omega} \frac{\partial u_{N}}{\partial n} v_{\Omega} \\
& =a\left(u_{N}-u, v_{\Omega}\right) \\
& \leq C\left\|u_{N}-u\right\|_{1}\left|v_{\Omega}\right|_{1} .
\end{aligned}
$$

Now, we consider $\left|v_{\Omega}\right|_{1}$. Using (35)-(36), we find for $\alpha>$ $1 / 2$ that

$$
\begin{aligned}
\left|v_{\Omega}\right|_{1}^{2}= & \left\|\nabla\left(\left(u_{N}-\Delta u_{N}\right) \Phi_{\Omega}^{\alpha}\right)\right\|_{0}^{2} \\
\leq & 2 \int_{\Omega} \Phi_{\Omega}^{2 \alpha}\left|\nabla\left(u_{N}-\Delta u_{N}\right)\right|^{2}+2 \int_{\Omega}\left|\nabla \Phi_{\Omega}^{\alpha}\right|^{2}\left(u_{N}-\Delta u_{N}\right)^{2} \\
\leq & C N^{2(2-\alpha)} \int_{\Omega} \Phi_{\Omega}^{\alpha}\left(u_{N}-\Delta u_{N}\right)^{2} \\
& +C \int_{\Omega} \Phi_{\Omega}^{2(\alpha-1)}\left(u_{N}-\Delta u_{N}\right)^{2} \\
\leq & C N^{2(2-\alpha)} \int_{\Omega} \Phi_{\Omega}^{\alpha}\left(u_{N}-\Delta u_{N}\right)^{2} \\
= & C N^{2(2-\alpha)}\left\|v_{\Omega} \Phi_{\Omega}^{-\alpha / 2}\right\|_{0}^{2},
\end{aligned}
$$

noticing that (35) is valid since the assumption $\alpha>1 / 2$ implies $2 \alpha-2>-1$, hence we take $\beta=\alpha, \alpha=2(\alpha-1)$ in (35) and the above third inequality holds. By the definition $\left\|v_{\Omega} \Phi_{\Omega}^{-\alpha / 2}\right\|_{0}=N \eta_{\alpha ; B}$, we obtain

$$
\eta_{\alpha ; B} \leq C N^{1-\alpha}\left\|u_{N}-u\right\|_{1} .
$$

To get an upper bound of the error indicator $\eta_{\alpha ; B}$ in the case of $0 \leq \alpha \leq 1 / 2$, using (35) we find for $\beta>1 / 2$ that

$$
\begin{aligned}
N \eta_{\alpha ; B} & =\left\|\left(u_{N}-\Delta u_{N}\right) \Phi_{\Omega}^{\alpha / 2}\right\|_{0} \\
& \leq C N^{\beta-\alpha}\left\|\left(u_{N}-\Delta u_{N}\right) \Phi_{\Omega}^{\beta / 2}\right\|_{0} \\
& \leq C N^{\beta-\alpha} \cdot N \cdot N^{1-\beta}\left\|u_{N}-u\right\|_{1} .
\end{aligned}
$$

Therefore,

$$
\eta_{\alpha ; B}^{2} \leq C N^{2(1-\alpha)}\left\|u_{N}-u\right\|_{1}^{2}
$$

We obtain the desired result immediately.

Lemma 10. Let $\alpha \in[0,1], \varepsilon>0$. Then there exists $C>0$ independent of $N$, such that

$$
\begin{aligned}
\eta_{\alpha ; E}^{2} \leq & C N^{\max \{1-2 \alpha+2 \varepsilon, 0\}} \\
& \times\left\{N\left\|u_{N}-u\right\|_{1}^{2}+\frac{1}{N}\left\|\lambda_{N} u_{N}-\lambda u\right\|_{0, \partial \Omega}^{2}\right\} .
\end{aligned}
$$

Proof. In order to obtain an upper bound for the error indicator $\eta_{\alpha ; E}$, we will use weight function and a proper extension operator. Let $e$ be an edge of $\Omega$; we construct a function $w_{e} \in H^{1}(\Omega)$ and $\left.w_{e}\right|_{e}=\left(\lambda_{N} u_{N}-\partial u_{N} / \partial n\right) \Phi_{e}^{\alpha}$.

Let $v_{e}=\left(\lambda_{N} u_{N}-\partial u_{N} / \partial n\right) \Phi_{e}^{\alpha}$. We extend $v_{e}$ to $\partial \Omega$ by using Lemma 6 where the polynomial $\pi_{N}$ corresponds to 
$\lambda_{N} u_{N}-\left(\partial u_{N} / \partial n\right)$, and from (37) we know that $v_{e}$ is zero on $\partial \Omega \backslash e$. We extend $w_{e}$ to $\partial \Omega$ such that $w_{e}=0$ on $\partial \Omega \backslash e$; then

$$
\begin{aligned}
\left\|\left(\lambda_{N} u_{N}-\frac{\partial u_{N}}{\partial n}\right) \Phi_{e}^{\alpha / 2}\right\|_{0, e}^{2}= & \int_{\partial \Omega}\left(\lambda_{N} u_{N}-\frac{\partial u_{N}}{\partial n}\right) w_{e} \\
= & \int_{\partial \Omega}\left(\lambda_{N} u_{N}-\lambda u\right) w_{e} \\
& +a\left(u-u_{N}, w_{e}\right) \\
& +\int_{\Omega}\left(u_{N}-\Delta u_{N}\right) w_{e} \\
\leq & \left\|\lambda_{N} u_{N}-\lambda u\right\|_{0, \partial \Omega}\left\|w_{e}\right\|_{0, \partial \Omega} \\
& +C\left\|u-u_{N}\right\|_{1}\left\|w_{e}\right\|_{1} \\
& +\left\|u_{N}-\Delta u_{N}\right\|_{0}\left\|w_{e}\right\|_{0} .
\end{aligned}
$$

Now we estimate $\left\|w_{e}\right\|_{0, \partial \Omega},\left\|w_{e}\right\|_{0},\left\|w_{e}\right\|_{1}$ in turn. First,

$$
\left\|w_{e}\right\|_{0, \partial \Omega}^{2} \leq C\left\|\left(\lambda_{N} u_{N}-\frac{\partial u_{N}}{\partial n}\right) \Phi_{e}^{\alpha / 2}\right\|_{0, e}^{2} .
$$

In the case of $\alpha \in(1 / 2,1]$ we have

$$
\begin{gathered}
\left\|w_{e}\right\|_{0}^{2} \leq C \varepsilon\left\|\left(\lambda_{N} u_{N}-\frac{\partial u_{N}}{\partial n}\right) \Phi_{e}^{\alpha / 2}\right\|_{0, e}^{2}, \\
\left\|w_{e}\right\|_{1}^{2} \leq C\left(\varepsilon N^{2(2-\alpha)}+\varepsilon^{-1}\right)\left\|\left(\lambda_{N} u_{N}-\frac{\partial u_{N}}{\partial n}\right) \Phi_{e}^{\alpha / 2}\right\|_{0, e}^{2} .
\end{gathered}
$$

Combining (58) and (59), we get

$$
\begin{aligned}
\left\|\left(\lambda_{N} u_{N}-\frac{\partial u_{N}}{\partial n}\right) \Phi_{e}^{\alpha / 2}\right\|_{0, e} \\
\leq C\left\|\lambda_{N} u_{N}-\lambda u\right\|_{0, \partial \Omega}+C\left[\varepsilon N^{2(2-\alpha)}+\varepsilon^{-1}\right]^{1 / 2} \\
\quad \times\left\|u-u_{N}\right\|_{1}+C \varepsilon^{1 / 2}\left\|u_{N}-\Delta u_{N}\right\|_{0} .
\end{aligned}
$$

By the definition of $\eta_{\alpha ; E}^{2}$ and setting $\alpha=0$ in Lemma 9, we have that

$$
\begin{aligned}
\eta_{\alpha ; E}^{2} \leq & C \frac{1}{N}\left\|\lambda_{N} u_{N}-\lambda u\right\|_{0, \partial \Omega}^{2} \\
& +C\left\{\frac{1}{N}\left[\varepsilon N^{2(2-\alpha)}+\varepsilon^{-1}\right]+N^{3} \varepsilon\right\}\left\|u-u_{N}\right\|_{1}^{2} .
\end{aligned}
$$

Setting $\varepsilon=1 / N^{2}$ yields the assertion for $\alpha>1 / 2$ in (61).

For the case $\alpha \in[0,1 / 2]$, setting $\beta=1 / 2+\varepsilon$ and using (35), we find

$$
\begin{aligned}
\eta_{\alpha ; E} & =\frac{1}{\sqrt{N}}\left\|\left(\lambda_{N} u_{N}-\frac{\partial u_{N}}{\partial n}\right) \Phi_{e}^{\alpha / 2}\right\|_{0, \partial \Omega} \\
& \leq C \frac{1}{\sqrt{N}} N^{\beta-\alpha}\left\|\left(\lambda_{N} u_{N}-\frac{\partial u_{N}}{\partial n}\right) \Phi_{e}^{\beta / 2}\right\|_{0, \partial \Omega} \\
& =C N^{\beta-\alpha} \eta_{\beta ; E} ;
\end{aligned}
$$

therefore,

$$
\eta_{\alpha ; E}^{2} \leq C N^{1+2 \varepsilon-2 \alpha} \eta_{\beta ; E}^{2}
$$

From the above discussion we complete the proof.

Combining Lemmas 9 and 10, we obtain the following theorem.

Theorem 11. Let $\alpha \in[0,1]$. Then there exists $C>0$ independent of $N$, such that

$$
\begin{aligned}
\eta_{\alpha}^{2} \leq & C N^{\max \{1-2 \alpha+2 \varepsilon, 0\}} \\
& \times\left\{N\left\|u_{N}-u\right\|_{1}^{2}+\frac{1}{N}\left\|\lambda_{N} u_{N}-\lambda u\right\|_{0, \partial \Omega}^{2}\right\} .
\end{aligned}
$$

Remark 12. The term $\left\|\lambda_{N} u_{N}-\lambda u\right\|_{0, \partial \Omega}^{2}$ is a term of higher order. In fact, for any $v \in H^{1}(\Omega)$, we have

$$
\begin{aligned}
\int_{\partial \Omega}( & \left.\lambda_{N} u_{N}-\lambda u\right) v \\
\quad & \int_{\partial \Omega} \lambda\left(u_{N}-u\right) v+\int_{\partial \Omega}\left(\lambda_{N}-\lambda\right) u_{N} v \\
& \leq\left(\lambda\left\|u_{N}-u\right\|_{0, \partial \Omega}+\left(\lambda_{N}-\lambda\right)\left\|u_{N}\right\|_{0, \partial \Omega}\right)\|v\|_{0, \partial \Omega} .
\end{aligned}
$$

Thus, taking $v=\lambda_{N} u_{N}-\lambda u$ and using the a priori estimates (23) and (25), we conclude that

$$
\begin{aligned}
\left\|\lambda_{N} u_{N}-\lambda u\right\|_{0, \partial \Omega} \leq & C N^{1-m-r / 2} \sup _{u \in V(\lambda),\|u\|_{1}=1}\|u\|_{m} \\
& +C\left\{N^{1-m} \sup _{u \in V(\lambda),\|u\|_{1}=1}\|u\|_{m}\right\}^{2} .
\end{aligned}
$$

\section{Spectral Method Based on the Shifted-Inverse Iteration}

High efficient finite element schemes have been researched further in recent years; for example, see [22, 25-31]. Based on these works, this paper combines the shifted-inverse iteration and spectral method to propose an efficient scheme.

Scheme 13 (spectral method based on the shifted-inverse iteration).

Step 1. Solve (13) in $S_{N_{1}}(\Omega)$ : Find $\lambda_{N_{1}} \in R, u_{N_{1}} \in S_{N_{1}}(\Omega)$, such that

$$
a\left(u_{N_{1}}, v\right)=\lambda_{N_{1}} b\left(u_{N_{1}}, v\right), \quad \forall v \in S_{N_{1}}(\Omega)
$$

Step 2. Solve a linear system in $S_{N_{2}}(\Omega)\left(N_{2}>N_{1}\right)$. Find $u^{N_{2}} \in$ $S_{N_{2}}(\Omega)$, such that

$$
a\left(u^{N_{2}}, v\right)-\lambda_{N_{1}} b\left(u^{N_{2}}, v\right)=b\left(u_{N_{1}}, v\right), \quad \forall v \in S_{N_{2}}(\Omega) .
$$

Step 3. Compute the Rayleigh quotient

$$
\lambda^{N_{2}}=\frac{a\left(u^{N_{2}}, u^{N_{2}}\right)}{b\left(u^{N_{2}}, u^{N_{2}}\right)} .
$$


TABLE 1: The eigenvalues of LGL spectral method.

\begin{tabular}{|c|c|c|c|c|}
\hline$N$ & DOF & $\lambda_{1}$ & $\lambda_{2}$ & $\lambda_{4}$ \\
\hline 3 & 16 & 0.24007936507936 & 1.49252393896074 & 2.08264705882353 \\
\hline 4 & 25 & 0.24007908542791 & 1.49230719085030 & 2.08264705882353 \\
\hline 5 & 36 & 0.24007908542792 & 1.49230316584831 & 2.08264705403320 \\
\hline 6 & 49 & 0.24007908542722 & 1.49230313468790 & 2.08264705403320 \\
\hline 7 & 64 & 0.24007908542723 & 1.49230313453462 & 2.08264705403320 \\
\hline 8 & 81 & 0.24007908542723 & 1.49230313453360 & 2.08264705403320 \\
\hline 9 & 100 & 0.24007908542722 & 1.49230313453358 & 2.08264705403318 \\
\hline 10 & 121 & 0.24007908542721 & 1.49230313453357 & 2.08264705403318 \\
\hline 11 & 144 & 0.24007908542722 & 1.49230313453359 & 2.08264705403321 \\
\hline 12 & 169 & 0.24007908542721 & 1.49230313453357 & 2.08264705403318 \\
\hline 13 & 196 & 0.24007908542723 & 1.49230313453360 & 2.08264705403323 \\
\hline 14 & 225 & 0.24007908542722 & 1.49230313453357 & 2.08264705403318 \\
\hline 15 & 256 & 0.24007908542724 & 1.49230313453359 & 2.08264705403320 \\
\hline 16 & 289 & 0.24007908542723 & 1.49230313453359 & 2.08264705403319 \\
\hline 17 & 324 & 0.24007908542728 & 1.49230313453361 & 2.08264705403321 \\
\hline 18 & 361 & 0.24007908542725 & 1.49230313453359 & 2.08264705403319 \\
\hline 19 & 400 & 0.24007908542721 & 1.49230313453356 & 2.08264705403317 \\
\hline 20 & 441 & 0.24007908542718 & 1.49230313453349 & 2.08264705403311 \\
\hline 21 & 484 & 0.24007908542705 & 1.49230313453341 & 2.08264705403305 \\
\hline 22 & 529 & 0.24007908542721 & 1.49230313453356 & 2.08264705403317 \\
\hline 23 & 576 & 0.24007908542727 & 1.49230313453364 & 2.08264705403326 \\
\hline 24 & 625 & 0.24007908542700 & 1.49230313453339 & 2.08264705403301 \\
\hline 25 & 676 & 0.24007908542723 & 1.49230313453356 & 2.08264705403317 \\
\hline 27 & 784 & 0.24007908542747 & 1.49230313453375 & 2.08264705403338 \\
\hline 29 & 900 & 0.24007908542735 & 1.49230313453373 & 2.08264705403335 \\
\hline 30 & 961 & 0.24007908542716 & 1.49230313453351 & 2.08264705403311 \\
\hline 31 & 1024 & 0.24007908542735 & 1.49230313453364 & 2.08264705403327 \\
\hline 34 & 1225 & 0.24007908542735 & 1.49230313453361 & 2.08264705403323 \\
\hline
\end{tabular}

Let $\left(\lambda_{N_{1}}, u_{N_{1}}\right)$ be the $k$ th eigenpair of (67) then $\left(\lambda^{N_{2}}, u^{N_{2}}\right)$ is regarded as the $k$ th approximate eigenpair of (13).

Theorem 14. Suppose that $V(\lambda) \subset H^{m}(\Omega)$ and $N_{1}$ is properly large. Let $\left(\lambda^{N_{2}}, u^{N_{2}}\right)$ be the $k$ th approximate eigenpair obtained by Scheme 13; then there exists the kth eigenpair $(\lambda, u)$ of (13), such that

$$
\begin{aligned}
& \left\|u^{N_{2}}-u\right\|_{1} \leq C\left(N_{1}^{-r+3(1-m)}+N_{2}^{1-m}\right), \\
& \left|\lambda^{N_{2}}-\lambda\right| \leq C\left(N_{1}^{-2 r+6(1-m)}+N_{2}^{2(1-m)}\right) .
\end{aligned}
$$

Proof. From the proof of Theorem 4.1 in [22] we can deduce that

$$
\begin{gathered}
\left\|u^{N_{2}}-u\right\|_{1} \leq C\left(\eta_{a}\left(N_{1}\right) \varepsilon_{N_{1}}^{3}+\varepsilon_{N_{2}}\right), \\
\left|\lambda^{N_{2}}-\lambda\right| \leq C\left(\eta_{a}\left(N_{1}\right)^{2} \varepsilon_{N_{1}}^{6}+\varepsilon_{N_{2}}^{2}\right),
\end{gathered}
$$

which together with Lemma 1 yields (70).

Theorem 14 tells us, when $N_{1}<N_{2} \leq N_{1}^{(r+3(m-1)) /(m-1)}$, the degree of precision (DOP) of $\lambda^{N_{2}}$ and $u^{N_{2}}$ obtained by Scheme 13 is $O\left(N_{2}^{2(1-m)}\right)$ and $O\left(N_{2}^{1-m}\right)$, respectively; that is to say, $\left(\lambda^{N_{2}}, u^{N_{2}}\right)$ have the same DOP with $\left(\lambda_{N_{2}}, u_{N_{2}}\right)$.

\section{Numerical Experiments}

Consider the Steklov eigenvalue problem (13) where $\Omega=$ $[0,1]^{2}$.

We use spectral method with tensor-product nodal basis at the LGL points (LGL spectral method) to solve (13). We construct two-dimensional basis functions by using the tensor product of one-dimensional basis functions. More precisely, let $\left\{h_{k}\right\}_{k=1}^{N+1}$ be the Lagrange basis polynomials associated with the LGL points; then $S_{N}(\Omega)=\operatorname{span}\left\{\phi_{i}: \phi_{i}=\right.$ $\left.h_{i 1}(x) h_{j 1}(y), i 1, j 1=1,2, \ldots, N+1, i=1,2, \ldots,(N+1)^{2}\right\}$, and let $u_{N}=\sum_{i} u_{i} \phi_{i}$; then (15) is equivalent to the following linear system:

$$
A U=\lambda_{N} B U
$$

where $A$ is called stiffness matrix, $B$ is called mass matrix, and

$$
\begin{gathered}
U=\left(u_{1}, u_{2}, \ldots, u_{(N+1)^{2}}\right)^{T}, \\
A=\left(a_{i, j}\right), \quad a_{i, j}=\int_{\Omega} \nabla \phi_{i} \cdot \nabla \phi_{j}+\int_{\Omega} \phi_{i} \phi_{j}, \\
1 \leq i, j \leq(N+1)^{2}, \\
B=\left(b_{i, j}\right), \quad b_{i, j}=\int_{\partial \Omega} \phi_{i} \phi_{j}, 1 \leq i, j \leq(N+1)^{2} .
\end{gathered}
$$


TABle 2: The eigenvalues of the bilinear finite element.

\begin{tabular}{lcccc}
\hline$h$ & DOF & $\lambda_{1}$ & $\lambda_{2}$ & $\lambda_{4}$ \\
\hline $1 / 8$ & 81 & 0.24022930645534 & 1.49577435506528 & 2.08269976804992 \\
$1 / 16$ & 289 & 0.24011662732054 & 1.49317640860702 & 2.08266035463158 \\
$1 / 32$ & 1089 & 0.24008847006616 & 1.49252179566534 & 2.08265038681122 \\
$1 / 64$ & 4225 & 0.24008143153493 & 1.49235782124229 & 2.08264788770449 \\
$1 / 128$ & 16641 & 0.24007967195002 & 1.49231680754965 & 2.08264726248054 \\
$1 / 150$ & 22801 & 0.24007951252139 & 1.49231309100913 & 2.08264720582238 \\
$1 / 200$ & 40401 & 0.24007932566556 & 1.49230873510836 & 2.08264713941521 \\
$1 / 256$ & 66049 & 0.24007923205310 & 1.49230655286905 & 2.08264710614529 \\
$1 / 300$ & 90601 & 0.24007919219220 & 1.49230562369281 & 2.08264709197866 \\
$1 / 400$ & 160801 & 0.24007914547718 & 1.49230453468660 & 2.08264707537565 \\
$1 / 512$ & 263169 & 0.24007912210763 & 1.49230398913452 & 2.08264706706984 \\
\hline
\end{tabular}

TABLE 3: The eigenvalues of shifted-inverse iterative method.

\begin{tabular}{lcccc}
\hline$N_{1}$ & $N_{2}$ & $\lambda_{1}^{N_{2}}$ & $\lambda_{2}^{N_{2}}$ & $\lambda_{4}^{N_{2}}$ \\
\hline 4 & 5 & 0.24007908542792 & 1.49230316584831 & 2.08264705403320 \\
4 & 6 & 0.24007908542722 & 1.49230313468790 & 2.08264705403320 \\
4 & 7 & 0.24007908542722 & 1.49230313453463 & 2.08264705403320 \\
4 & 8 & 0.24007908542723 & 1.49230313453360 & 2.08264705403320 \\
\hline
\end{tabular}

We program with Matlab to solve (72), and the numerical results are presented in Table 1.

In the tables in this section, DOF denotes the degree of freedom (number of nodes).

From Table 1 we can see that

$$
\lambda_{1} \approx 0.240079085427
$$

$\lambda_{2}=\lambda_{3} \approx 1.492303134533, \quad \lambda_{4} \approx 2.082647054033$.

We also use the bilinear finite element to solve (13), and the numerical results are listed in Table 2.

From Table 2 we see that

$$
\begin{gathered}
\lambda_{1} \approx 0.2400791, \quad \lambda_{2}=\lambda_{3} \approx 1.49230, \\
\lambda_{4} \approx 2.0826470 .
\end{gathered}
$$

We use Scheme 13 to solve (13), and let $\lambda_{i}^{N_{2}}$ be the $i$ th approximate eigenvalue $(i=1,2,4)$, and the numerical results are presented in Table 3.

From Table 3 we find the eigenvalues obtained by Scheme 13 have the same accuracy with $\lambda_{i, N_{2}}$ which is computed directly in $S_{N_{2}}(\Omega)$ by LGL spectral method.

Since we do not know the exact eigenvalues of the Steklov eigenvalue problem, many research studies reported the approximate eigenvalues; for example, see $[15,22]$. This paper uses the LGL spectral method to solve (13), and in Table 1 we give

$$
\lambda_{1} \approx 0.240079085427 \text {, }
$$

$$
\lambda_{2}=\lambda_{3} \approx 1.492303134533, \quad \lambda_{4} \approx 2.082647054033 \text {. }
$$

We think these approximate eigenvalues have high accuracy.

\section{Acknowledgment}

This work is supported by the National Natural Science Foundation of China (Grant no. 11201093).

\section{References}

[1] C. Conca, J. Planchard, and M. Vanninathan, Fluids and Periodic Structures, vol. 38, John Wiley \& Sons, New york, NY, USA, 1995.

[2] D. Bucur and I. R. Ionescu, "Asymptotic analysis and scaling of friction parameters," Zeitschrift für Angewandte Mathematik und Physik, vol. 57, no. 6, pp. 1042-1056, 2006.

[3] S. Bergman and M. Schiffer, Kernel Functions and Elliptic Differential Equations in Mathematical Physics, Academic Press, New York, NY, USA, 1953.

[4] A. Bermúdez, R. Rodríguez, and D. Santamarina, "A finite element solution of an added mass formulation for coupled fluid-solid vibrations," Numerische Mathematik, vol. 87, no. 2, pp. 201-227, 2000.

[5] H. J. Ahn, "Vibrations of a pendulum consisting of a bob suspended from a wire: the method of integral equations," Quarterly of Applied Mathematics, vol. 39, no. 1, pp. 109-117, 1981.

[6] D. B. Hinton and J. K. Shaw, "Differential operators with spectral parameter incompletely in the boundary conditions," Funkcialaj Ekvacioj, vol. 33, no. 3, pp. 363-385, 1990.

[7] J. H. Bramble and J. E. Osborn, "Approximation of Steklov eigenvalues of non-selfadjoint second order elliptic operators," in The Mathematical Foundations of the Finite Element Method with Applications to Partial Differential Equations, A. K. Aziz, Ed., Academic Press, San Diego, Calif, USA, 1972.

[8] A. B. Andreev and T. D. Todorov, "Isoparametric finite-element approximation of a Steklov eigenvalue problem," IMA Journal of Numerical Analysis, vol. 24, no. 2, pp. 309-322, 2004. 
[9] M. G. Armentano and C. Padra, "A posteriori error estimates for the Steklov eigenvalue problem," Applied Numerical Mathematics, vol. 58, no. 5, pp. 593-601, 2008.

[10] A. Alonso and A. D. Russo, "Spectral approximation of variationally-posed eigenvalue problems by nonconforming methods," Journal of Computational and Applied Mathematics, vol. 223, no. 1, pp. 177-197, 2009.

[11] Y. Yang, Q. Li, and S. Li, "Nonconforming finite element approximations of the Steklov eigenvalue problem," Applied Numerical Mathematics, vol. 59, no. 10, pp. 2388-2401, 2009.

[12] Q. Li, Q. Lin, and H. Xie, "Nonconforming finite element approximations of the Steklov eigenvalue problem and its lower bound approximations," Applications of Mathematics, vol. 58, no. 2, pp. 129-151, 2013.

[13] Q. Li and Y. Yang, "A two-grid discretization scheme for the Steklov eigenvalue problem," Journal of Applied Mathematics and Computing, vol. 36, no. 1-2, pp. 129-139, 2011.

[14] H. Bi and Y. Yang, "A two-grid method of the non-conforming Crouzeix-Raviart element for the Steklov eigenvalue problem," Applied Mathematics and Computation, vol. 217, no. 23, pp. 9669-9678, 2011.

[15] M. Li, Q. Lin, and S. Zhang, "Extrapolation and superconvergence of the Steklov eigenvalue problem," Advances in Computational Mathematics, vol. 33, no. 1, pp. 25-44, 2010.

[16] W. Tang, Z. Guan, and H. Han, "Boundary element approximation of Steklov eigenvalue problem for Helmholtz equation," Journal of Computational Mathematics, vol. 16, no. 2, pp. 165$178,1998$.

[17] L. Cao, L. Zhang, W. Allegretto, and Y. Lin, "Multiscale asymptotic method for Steklov eigenvalue equations in composite media," SIAM Journal on Numerical Analysis, vol. 51, no. 1, pp. 273-296, 2013.

[18] C. Canuto, M. Y. Hussaini, A. Quarteroni, and T. A. Zang, Spectral Methods Evolution to Complex Geometries and Applications to Fluid Dynamics, Springer, Berlin, Germany, 2007.

[19] J. Shen, T. Tang, and L.-L. Wang, Spectral Methods Algorithms, analysis and applications, vol. 41, Springer, Heidelberg, Germany, 2011.

[20] I. Babuska and J. Osborn, "Eigenvalue Problems," in Handbook of Numerical Analysis, Finite Element Methods (Part 1), P. G. Ciarlet and J. L. Lions, Eds., vol. 2, pp. 640-787, Elsevier Science Publishers, 1991.

[21] J. M. Melenk and B. I. Wohlmuth, "On residual-based a posteriori error estimation in $h p$-FEM," Advances in Computational Mathematics, vol. 15, no. 1-4, pp. 311-331, 2001.

[22] Y. Yang and H. Bi, "Two-grid finite element discretization schemes based on shifted-inverse power method for elliptic eigenvalue problems," SIAM Journal on Numerical Analysis, vol. 49, no. 4, pp. 1602-1624, 2011.

[23] Y. Yang, Finite Element Methods for Eigenvalue Problems, Science Press, Beijing, China, 2012.

[24] J. M. Melenk, " $h p$-interpolation of nonsmooth functions and an application to $h p$-a posteriori error estimation," SIAM Journal on Numerical Analysis, vol. 43, no. 1, pp. 127-155, 2005.

[25] Y. Yang and W. Jiang, "Upper spectral bounds and a posteriori error analysis of several mixed finite element approximations for the Stokes eigenvalue problem," Science China Mathematics, vol. 56, no. 6, pp. 1313-1330, 2013.

[26] J. Hu and Y. Huang, "The correction operator for the canonical interpolation operator of the Adini element and the lower bounds of eigenvalues," Science China Mathematics, vol. 55, no. 1, pp. 187-196, 2012.
[27] A. Naga and Z. Zhang, "Function value recovery and its application in eigenvalue problems," SIAM Journal on Numerical Analysis, vol. 50, no. 1, pp. 272-286, 2012.

[28] Z. Si, Y. He, and K. Wang, "A defect-correction method for unsteady conduction convection problems I: spatial discretization," Science China Mathematics, vol. 54, no. 1, pp. 185-204, 2011.

[29] Y. Yang, W. Jiang, Y. Zhang, W. J. Wang, and H. Bi, "A twoscale discretization scheme for mixed variational formulation of eigenvalue problems," Abstract and Applied Analysis, vol. 2012, Article ID 812914, 29 pages, 2012.

[30] Y. Yang, Y. Zhang, and H. Bi, "Multigrid discretization and iterative algorithm for mixed variational formulation of the eigenvalue problem of electric field," Abstract and Applied Analysis, vol. 2012, Article ID 190768, 25 pages, 2012.

[31] H. Bi and Y. Yang, "Multiscale discretization scheme based on the Rayleigh quotient iterative method for the Steklov eigenvalue problem," Mathematical Problems in Engineering, vol. 2012, Article ID 487207, 18 pages, 2012. 


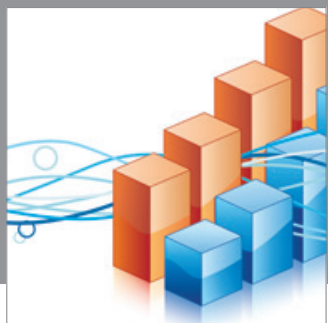

Advances in

Operations Research

mansans

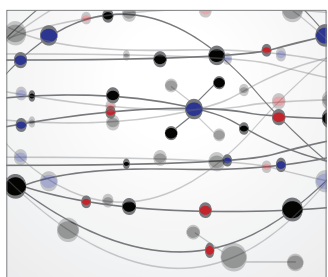

The Scientific World Journal
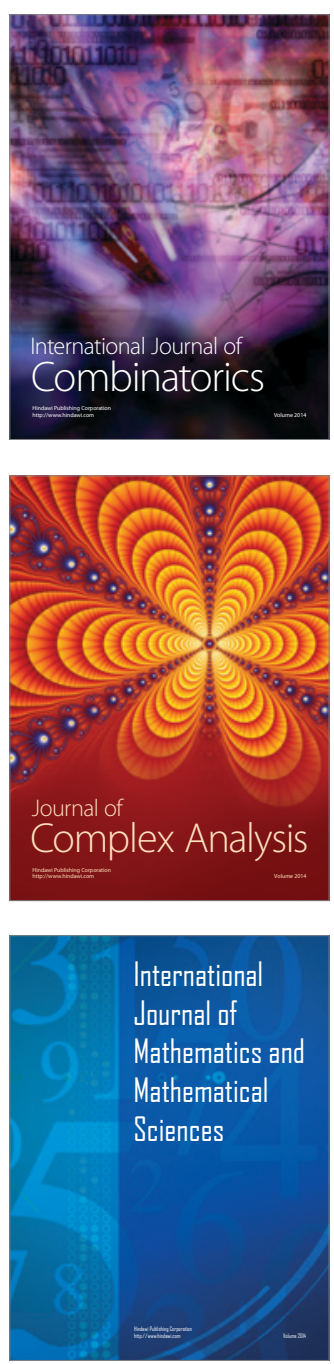
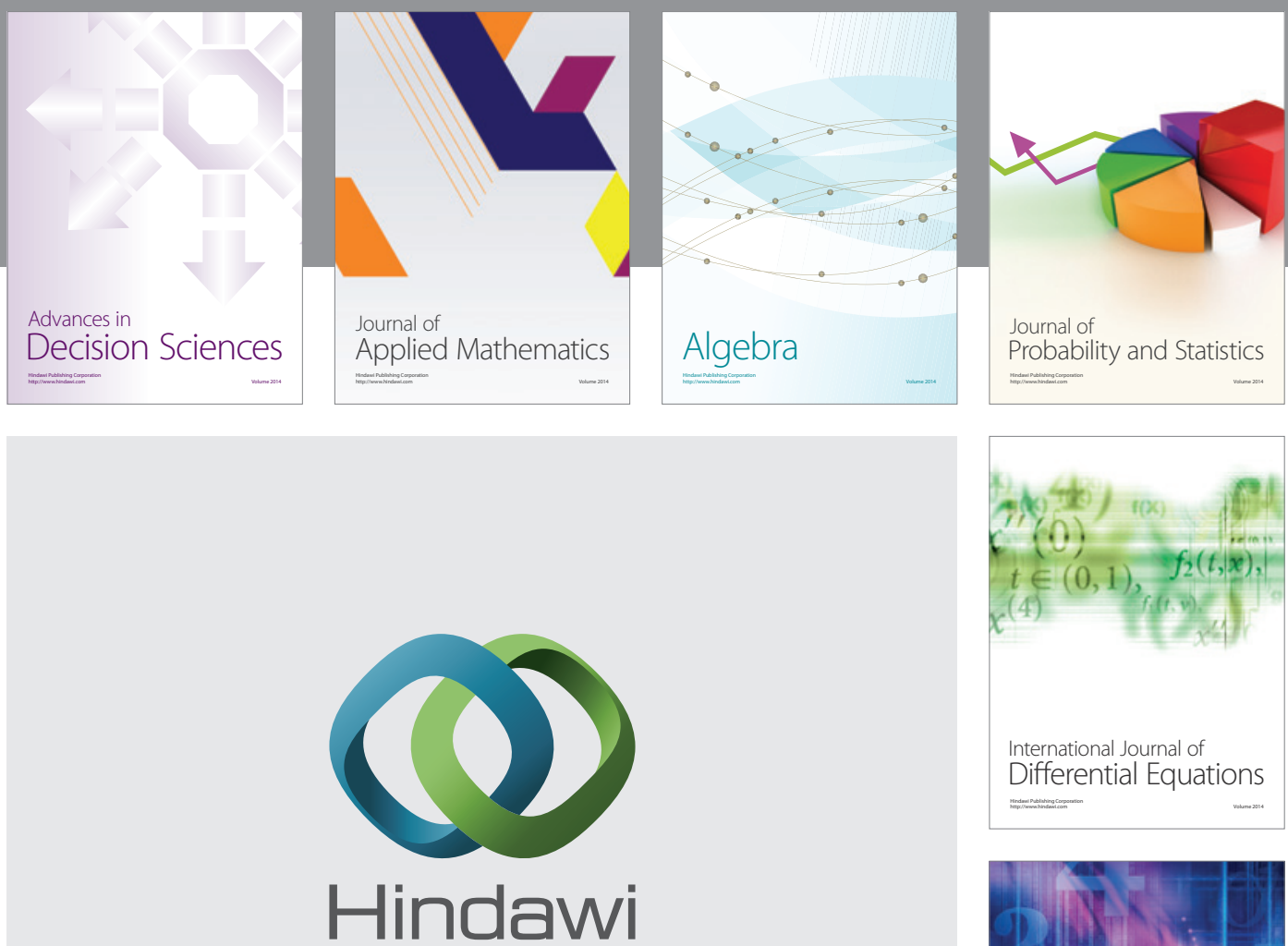

Submit your manuscripts at http://www.hindawi.com
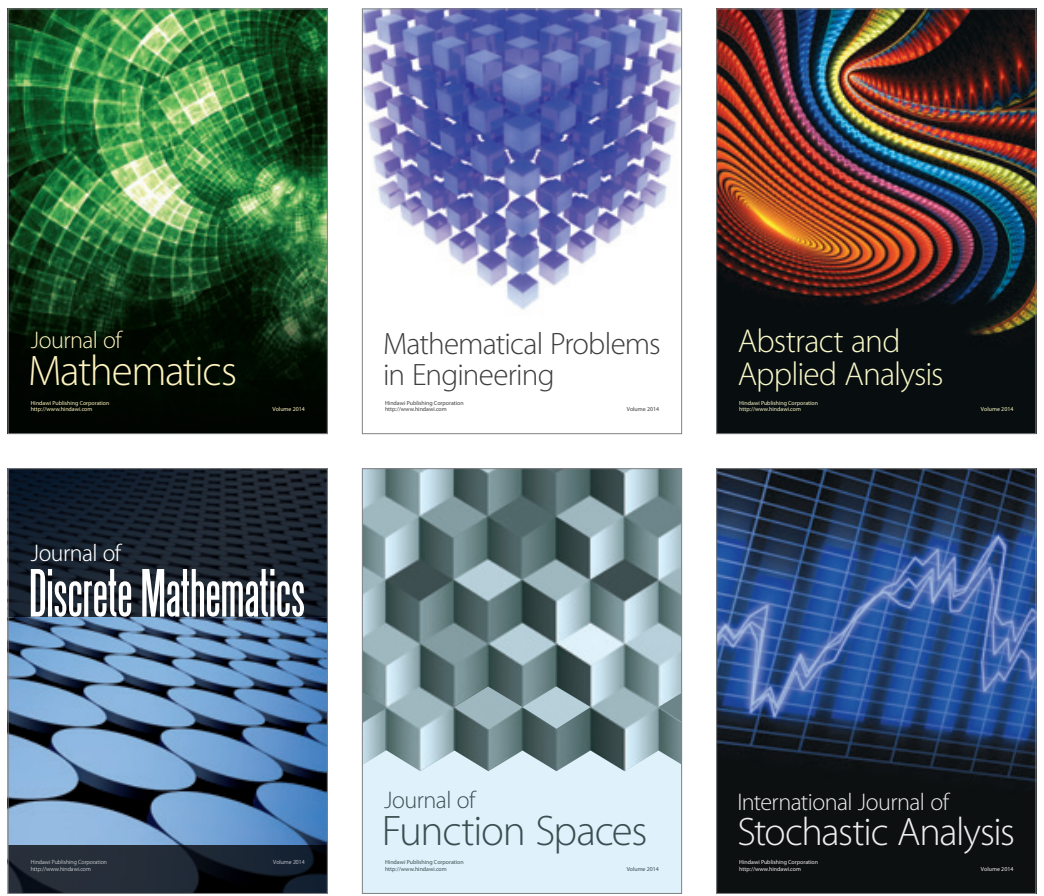

Journal of

Function Spaces

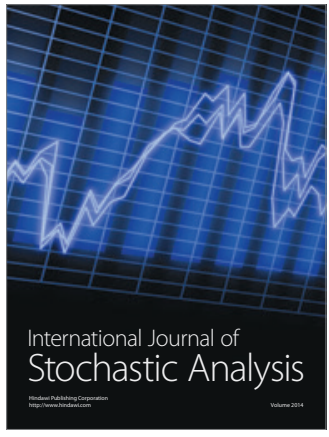

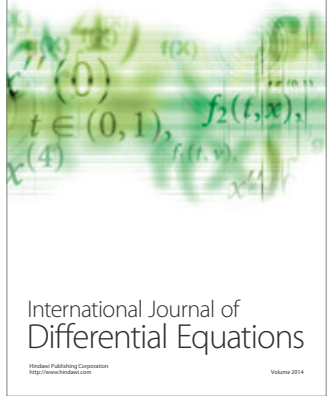
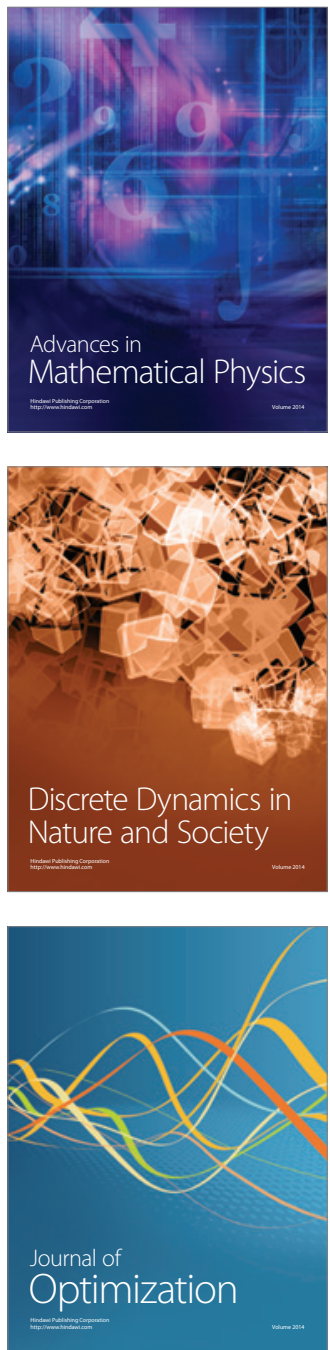